\title{
Una mirada desde la percepción del cuerpo de la
}

\section{persona mayor}

\author{
Eliana Monsalve-Jaramillo ${ }^{1}$ (D) , Claudia Inés Bohórquez-Olaya' ${ }^{1}$, Elisa Andrea Cobo-Mejía ${ }^{1}$
}

\begin{abstract}
RESUMEN
Introducción: El concepto de cuerpo envejecido proviene de la representación social y el estereotipo preconcebido de cada persona acerca de la vejez, el cual, a pesar de ser un proceso natural del ciclo vital, generalmente se ve como negativo.

Objetivo: Comprender la percepción del cuerpo envejecido que tienen las personas mayores de la ciudad de Tunja y de qué forma ayuda a construir la estética en la vejez.

Métodos: Investigación cualitativa, con enfoque hermenéutico. Se llevaron a cabo cinco grupos focales con la participación de dieciocho personas mayores. Para el análisis se empleó el programa Atlas.ti, con el reconocimiento de códigos, categorías y familias.

Resultados: Se reconoció cómo la percepción del cuerpo se vincula con los cambios físicos y psicológicos, en que la aceptación de la vejez se asume desde la resignación; adicionalmente, el adulto emplea prácticas de autocuidado y embellecimiento, con la finalidad de mitigar los cambios y propender hacia una estética que se acompaña de funcionalidad e independencia.

Conclusiones: Se logró evidenciar que la percepción que tienen las personas mayores a cerca de su cuerpo envejecido y su estética están dadas desde la reelaboración y reconfiguración del concepto proporcionado por el paso de los años.
\end{abstract}

Palabras clave: cuerpo humano; envejecimiento; belleza; cultura de la belleza; estética.

${ }^{1}$ Universidad de Boyacá, Tunja, Colombia.

Autora de correspondencia: Elisa Andrea Cobo-Mejía. Correo electrónico: eacobo@uniboyaca.edu.co

Citar este artículo así:

Monsalve-Jaramillo E, Bohórquez-Olaya Cl, Cobo-Mejía EA. Una mirada desde la percepción del cuerpo de la persona mayor. Rev Investig Salud Univ Boyacá. 2021;8(1):33-47. https://doi.org/10.24267/23897325.598 


\title{
A Look from the Perception of the Elderly Person's Body
}

\begin{abstract}
Introduction: The concept of the aging body comes from the social representation and the preconceived stereotype of each person about old age, which despite being a natural process of the life cycle is generally seen as negative.
\end{abstract}

Objective: To understand the perception of the aging body that older people in the city of Tunja have, and how to improve their aesthetics in old age.

Methods: Qualitative research, with a hermeneutical approach, five focus groups were carried out with the participation of eighteen elderly people, for the analysis the Atlas.ti program was used with the recognition of codes, categories and families.

Results: It was recognized as the perception of the body, it is related to changes in the physical and psychological, where the acceptance of old age is assumed from resignation, additionally, the adult uses self-care and beautification practices, in order to mitigate the changes and strive for aesthetics that is accompanied by functionality and independence.

Conclusions: It was possible to show that the perception that older people have about their aging body and its aesthetics are given from the re-elaboration and reconfiguration of the concept provided by the passage of time.

Keywords: human body; aging; beauty; culture of beauty; aesthetics. 


\title{
Um olhar desde a percepção do corpo do idoso
}

\begin{abstract}
RESUMO
Introdução: O conceito de corpo envelhecido provém da representação social do estereótipo preconcebido de cada pessoa sobre a velhice, que, mesmo sendo um processo natural do ciclo de vida, geralmente è enxergado como negativo.
\end{abstract}

Objetivo: Compreender a percepção do corpo envelhecido da população idosa da cidade de Tunja e de qual forma contribui na construção da estética na velhice.

Métodos: Pesquisa qualitativa, com foco hermenêutico. Foram realizados cinco grupos focais com a participação de dezoito idosos. Foi utilizado o programa Atlas.ti para a analises de dados, com o reconhecimento de códigos, categorias e famílias.

Resultados: Foi reconhecido que a percepção do corpo esta ligada com as mudanças físicas e psicológicas, em que a aceitação da velhice é assumida a partir da resignação: além disso, o idoso utiliza praticas de autocuidado e embelezamento, a fim de amenizar as mudanças e tender a uma estética acompanhada de funcionalidade e independência.

Conclusões: Foi possível evidenciar que as percepções que têm as pessoas idosas sobre seu corpo envelhecido e sua estética se dão a partir da reelaboração e reconfiguração do conceito proporcionado pelo passar dos anos.

Palavras-chave: corpo humano; envelhecimento; beleza; cultura da beleza; estética 


\section{INTRODUCCIÓN}

El cuerpo sufre cambios relacionados con la forma en que el ser humano lo concibe y emplea; así, ya a mediados del siglo $\mathrm{XX}$, el cuerpo es un tema de interés desde el consumismo, que promueve formas de lo bello y saludable y que lo convierten en objeto de culto (1). Luego, el cuerpo se convierte en un objeto estético que debe responder a estereotipos, determinados en función del género, la edad, la raza, la condición social, la ocupación, entre otras.

De ahí que el cuerpo de las personas mayores publicitado en comerciales sea representado desde una imagen negativa, relacionada con las arrugas, la lentitud del movimiento y la falta de energía, que responde a estereotipos fundados culturalmente y que lo puede llevar a presentar problemas psicológicos, como depresión y ansiedad, asociadas con una mayor insatisfacción con la imagen corporal, que se caracteriza además por la modificación de la autoimagen (2).

En ese orden de ideas, la estética moderna de lo bello se relaciona con la estética de lo terso (3), que no admite rugosidades, en la cual el cuerpo debe ser liso sin superficies ásperas que lo harán bello visualmente. Incluso, Byung-Chul Han refiere cómo, desde esta estética, la robustez y la fortaleza merman frente a la ternura y finura, que se consideran propiedades bellas: "el cuerpo es fino cuando consta de partes tersas que no muestran aspereza ni confunden la vista. El cuerpo bello que suscita amor y alegría no hace esperar resistencia" (3). Concluye que toda aspereza estropea la belleza; entonces, como aspereza se entenderían las arrugas y el aumento del peso corporal, cambios que suelen suceder en la vejez e inciden en la concepción de lo bello.

Dado lo anterior, la vejez se construye a partir de la experiencia y las interacciones que van a crear formas de comprender, en este caso de ser vieja o viejo. Según María del Pilar Duque Duque: "las representaciones constituyen modelos explicativos que le permiten a un grupo interpretar las experiencias propias y de los otros" (4), que configuran lo que se entiende por estética en esta etapa de la vida, en la cual varios de los estereotipos sociales presentes sobre cuerpo, belleza y juventud dan paso a unos nuevos que, por lo general, no son positivos, sino negativos, y como expone Duque Duque, se rodean de discriminación.

La influencia que esto ejerce en la autoimagen que tiene de sí misma la persona mayor es trascendental, por cuanto, a pesar de que el envejecimiento es un proceso individual y depende de factores como la edad cronológica, la edad biológica, la edad psicológica y la edad funcional, se ve seriamente afectada, al punto de asociar su proceso natural de envejecimiento con enfermedad, incapacidad, dependencia, improductividad, factores que afectan 
seriamente su vida emocional y la predisponen a la tristeza, la melancolía y la depresión (5).

En este sentido, los estereotipos negativos son, por lo general, perjudiciales para la relación con las personas mayores, ya que favorecen la estigmatización y otorgan a esta etapa de la vida un carácter negativo, en la cual "la juventud se desvanece y la belleza es fugaz" (6). Por esta razón, surgen los siguientes interrogantes: cómo se piensa ese cuerpo estético en la vejez, qué propiedades tiene, qué imagen proyecta. Luego el objeto de este texto es vislumbrar la percepción del cuerpo envejecido de las personas mayores de la ciudad de Tunja (Colombia), para comprender su autopercepción corporal en esta etapa de la vida.

\section{MATERIALES Y MÉTODOS}

Esta fue una investigación cualitativa con enfoque hermenéutico, en la cual dos investigadores con experiencia previa en técnicas cualitativas llevaron a cabo cinco grupos focales con dieciocho personas mayores hasta lograr la saturación teórica, que estuvo dada por las características de los participantes, en que para la mayoría la imagen corporal a esta edad no era un factor determinante y valoraban más la salud y la independencia. Como criterios de inclusión se tuvieron en cuenta la ubicación en la zona urbana de Tunja (Colombia), el estrato socioeconómico y la técnica de muestreo, que fue no probabilística por cuotas secuencial. Los participantes eran provenientes de centros de actividad física y comunidad, en general. Se excluyeron aquellos quienes tuvieran dificultades auditivas y cognitivas. En la investigación se plantearon categorías inductivas presentadas en la tabla 1.

Así, se trabajó con los grupos focales empleando preguntas guía, a partir de las cuales se profundizaba según las respuestas dadas. Además, se modificaron y adaptaron a medida que se iban realizando. Los grupos focales se grabaron y tuvieron una duración de 40 a 60 minutos, para posteriormente transcribirlos, con el consecuente análisis de los contenidos desde la codificación hasta la categorización de los datos encontrados por dos investigadores. En este proceso se utilizó el software Atlas.ti, versión 8.0, y con la información contenida en este se inició la creación de las redes semánticas en las que se incluyeron las categorías, las subcategorías y los códigos que la sustentan.

Por último, esta fue una investigación sin riesgo, según la Resolución 008430 de 1993, que contó con aval del Comité de Ética de la Universidad de Boyacá.

\section{RESULTADOS}

Las personas mayores participantes tenían una media de 72 años de edad, de las cuales el $88 \%$ eran mujeres, pensionadas o dedicadas al hogar. Una de ellas estaba activa laboralmente. La 
Tabla 1. Categorías de análisis

\begin{tabular}{ll}
\hline \multicolumn{1}{c}{ Categorías } & \multicolumn{1}{c}{ Subcategorías } \\
\hline Envejecer & $\begin{array}{l}\text { Autonomía: se refiere a la toma de decisiones y manejo del tiempo libre, cuando la familia (pareja/ hijos/ nie- } \\
\text { tos) no se vean involucrados. }\end{array}$ \\
& $\begin{array}{l}\text { Funcionalidad: se refiere a lo funcional que es en sus vidas cotidianas, tanto en la casa como en la calle. } \\
\text { Sociedad: describe la imagen y percepción acerca de la sociedad de cada uno. } \\
\text { Cambios de estilo de vida: muestra cómo se generan cambios tanto en sus actitudes y aptitudes físicas. }\end{array}$ \\
\hline $\begin{array}{l}\text { Autopercepción del } \\
\text { cuerpo envejecido }\end{array}$ & $\begin{array}{l}\text { Autopercepción: muestra cómo cada uno se ve a sí mismo en la actualidad. } \\
\text { Percepción del otro: muestra cómo cada uno ve a el otro en la actualidad. }\end{array}$ \\
\hline Aceptación de la vejez: se refiere a la aceptación y proceso de envejecimiento llevado por la familia. \\
Rol familiar & $\begin{array}{l}\text { Motivaciones: se refiere al logro de metas a pesar de la edad y las motivaciones externas, como son los hijos o } \\
\text { los nietos. } \\
\text { Cambios en la calidad de vida: muestra cómo el envejecimiento ha generado cambios en su calidad de vida. }\end{array}$ \\
& $\begin{array}{l}\text { Dinámica familiar en el tiempo libre: da cuenta de las dinámicas familiares los fines de semana o en el tiempo } \\
\text { libre. }\end{array}$ \\
\hline $\begin{array}{l}\text { Relaciones con pares: Se refiere a la relación directa que existe con pares y cómo por medio de esta se constru- } \\
\text { yen vínculos cercanos. } \\
\text { Relaciones interperso- } \\
\text { Dales y amistad }\end{array}$ & \begin{tabular}{l} 
mente en sus vidas. \\
\hline
\end{tabular}
\end{tabular}

mayoría de los participantes pertenecía al estrato socioeconómico 3. A continuación, se describen los códigos y las categorías emergentes del estudio. Con relación a los códigos se identifican 21 , conectados con la categoría de estética del cuerpo, los cuales exponen ideas como envejecer, autonomía, autocuidado, autopercepción, aceptación entre otros (figura 1).

\section{Envejecer}

Esta categoría se determinó a partir de las percepciones que tienen las personas mayores acerca del proceso de envejecer: manifiestan que es parte del ciclo de la vida y es cuando descubren diferentes cambios en lo físico y en lo psicológico; además, en las dimensiones capacidad física, capacidad funcional, apariencia física y autoconcepto físico general. Una de las personas mayores lo manifiesta: "Pues terminar ya su ciclo de vida porque uno nace crece se reproduce y muere..." (GF4. P1; 1:50). Ahora bien, otro percibe que envejecer se convierte en una etapa de maduración en todo sentido. "Envejecer es otra etapa de la vida y que ya es la madurez de nuestra existencia" (GF4. P2; 1:51). Por otro lado, esta categoría es 
Figura 1. Árbol de códigos

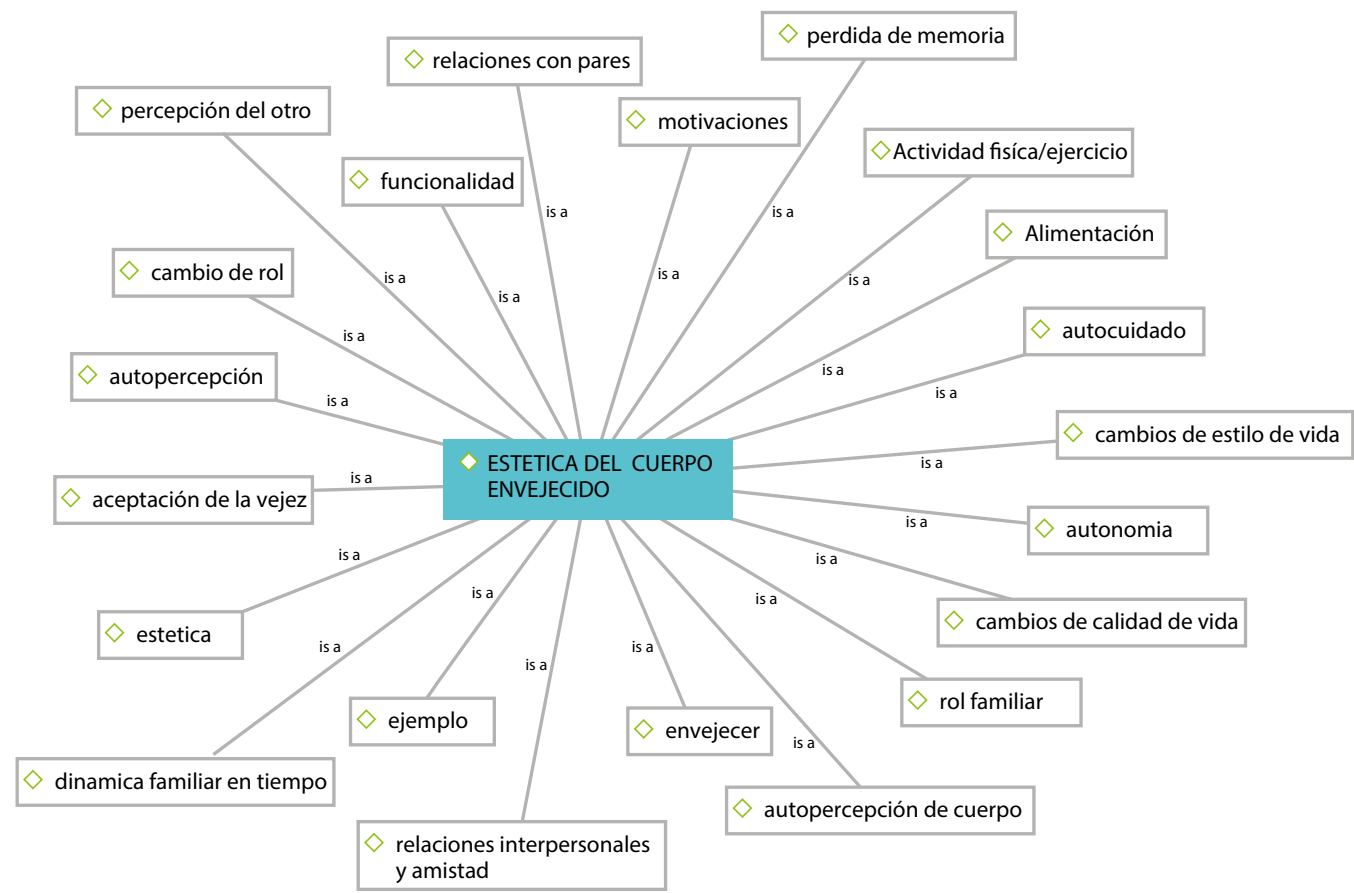

definida desde la enfermedad y las cohibiciones por ejemplo llega la etapa de la vejez, primeramente, que trae consigo esta nueva etapa, evidente en la siguiente: "Bueno, envejecer, creo que es cuando uno ya tiene la etapa de cambio de vida que uno se da cuenta de tantas cosas que empiezan a salir a flote enfermedades antes era como tan jovial y bueno y es a raíz del envejecimiento del cambio de vida se notan muchos cambios..." (GF3. P2; 1:38). Otra afirmación es: "Lo que pasa es que cuando uno envejece el metabolismo cambia, todo es $\tan$ diferente eso no es tan sencillo..." (GF1. E1; 1:22). "Pues envejecer significa que ya las arrugas, la tensión alta, los triglicéridos" (GF2. P1; 1:27).

\section{Prácticas de autocuidado}

Al revisar esta red semántica se observó que está construida por subcategorías como cuidado y actividad física/ejercicio. Tales prácticas de autocuidado ayudan a las personas mayores a mitigar los cambios psicológicos y físicos que tiene el proceso de envejecimiento. El descuido de su parte física 
lo ven como una condición que hace desmejorar su imagen; por eso, es importante para algunos de ellos practicarlas. Ello se evidencia en la siguiente expresión: "Cuando más tiene uno que hacer actividad, porque entre más años tenga uno, más actividad, compensar" (GF1. P3; 1:1). Así es como cuidarse se convierte en una parte importante en esta etapa del ciclo vital: "Llegar a la vejez pues es cuidarse uno, por ejemplo, que a esta edad que tenemos, de salir a hacer ejercicios, cuidarnos en la comidita, hacer nuestra vida, progresar, salir adelante..." (GF1. P2; 1:2). Estas prácticas de autocuidado hacen que las personas mayores se sientan bien: "Hacer ejercicio y mirarse bueno así estoy viejita, pero me siento bien" (GF1. P4; 1:20), ya que sienten que pueden realizar diferentes actividades que a pesar de su edad no creen que podrían hacerlas: "Desde que uno este alentado uno puede hacer cualquier actividad, claro que no demasiada pesada, pero si puede uno bailar, hacer deporte desde que a uno no le duela nada, pero si uno tiene un dolor físico eso si le impide" (GF3. P1; 1:47).

\section{Cambios de calidad de vida}

Al llegar a esta etapa de la vida, se evidencia el cambio en la calidad de vida de las personas mayores, teniendo en cuenta que esta se relaciona con las dimensiones, físicas, psicológicas y ambientales que posibilitan el desempeño de la persona en los ámbitos individual y comunitario que, en consecuencia, es dependiente de la funcionalidad y capacidad física. Desde este punto, la calidad de vida es vista desde otra perspectiva y también se siente diferente, afirmando que existe: "Pérdida de la ligereza en las actividades" (GF3. P3; 1:39). "Como pierde fuerza, agilidad y perder la lucidez de los órganos" (GF3. P1; 1:40). Estas pérdidas descritas hacen que los comportamientos y conductas de las personas cambien, afectando notablemente su calidad de vida. Particularmente, una persona afirma que: "A mí me afecta porque me canso mucho; antes no, en cambio ahorita por lo gordita no puede hacer lo mismo, correr, brincar; ya le toca a uno hacer más suavecito las cosas... En cambio, más joven pues uno brincaba y hacía, pero ya ahorita a mediado que van llegando las cosas le toca a uno más quietico..." (GF1. P1; 1:63).

\section{Prácticas de embellecimiento}

Se entiende por estas, todas y cada una de las prácticas que las personas realizan para verse físicamente mejor $y$, asimismo, para sentirse mejor. Están relacionadas con arreglarse, que se entienden desde su perspectiva como: "Entre más uno viva limpio y arreglado mejor..." (GF2. P4; 1:64). Esta apreciación es vista desde ambos sexos, pero en el caso de las mujeres las prácticas de embellecimiento son más elaboradas, así como una de ellas lo afirma: "Debe tratar uno de arreglarse, que bueno, si no se sabe pintar lo labios, pues 
aprenda a pintárselos, que los ojos que las cejas que se lo trate principalmente de lo personal..." (GF2. P3; 1:34). Por otro lado, estas prácticas de embellecimiento se ven reflejadas en los cuidados del cuerpo, en aspectos como la alimentación y la realización de ejercicio, donde también afirman que son importantes los buenos hábitos para mantenerse estéticamente bien. En términos generales, esto da cuenta de una buena imagen corporal, lo que es claro en el siguiente apartado: "Voy mucho a natación, me gustan mucho las aguas termales son muy beneficiosas para el cuerpo. Contiene demasiadas minerales calcio. Tiene magnesio, tiene cloruro, tiene azufre; entonces eso es muy beneficioso para la piel..." (GF4. P2; 1:57). "Ahora todo es ensaladitas y todo eso... sí light" (GF1. P1; 1:62).

\section{Autopercepción del cuerpo}

La autopercepción del cuerpo envejecido la describen como los cambios físicos que aparecen en el cuerpo con el paso de los años: la piel arrugada, las pecas, es decir, los cambios ocurridos más representativos. Ello es producto de los procesos de adaptación y aceptación a cambios normales de la edad, sin desconocer la importancia del autocuidado para el mantenimiento del cuerpo y su función. Entonces, una mujer afirma que: "Le salen pequitas en las manos, en la cara, empieza uno a perder toda la belleza..." (GF3. P1; 1:44). Se empiezan también a redefinir conceptos como lo bello y lo feo, donde la belleza se asocia con cuerpos funcionales e independientes, sin desconocer sobre todo en las mujeres la importancia que le dan a las prácticas de embellecimiento: "Claro, sí, porque a uno le va a dar tristeza porque ya no va a estar tan bonito como antes, aunque uno se considera que todavía uno no va a estar tan feo" (GF3. P1; 1:46). "Los cambios propios de la edad... pero el cuerpo así envejezca... Es bonito" (GF1. E1; 1:18). Y aunque las personas mayores son conscientes de que su cuerpo cambió, y este hecho hace que su autoconcepto varié, también es visto que su sentir no ha cambiado, como lo afirma uno de ellos: "Se cambia la belleza, pero el corazón sigue siendo el mismo" (GF3. P1; 1:45).

\section{Aceptación de la vejez}

La aceptación de la vejez se comunica como una resignación ante la etapa que se está viviendo, en que la persona mayor entiende que debe aceptar este cambio para así poder vivir sin tener que añorar su juventud diariamente. Así es como lo afirman algunos de ellos: "Si ya le toca a uno ver que ya la edad va pasando, entonces le toca a uno resignarse, tratar cuidarse uno lo más posible para aguantar la vejez" (GF1. P4; 1:7). "Uno debe aceptarse como es, no lo que los demás digan, aceptarse como es no ponerse en qué dirán que me arrugué no yo me acepto como soy" (GF2. P4; 1:33). "... si porque ya lo que fue, fue... Ya nos toca es cuidarnos a no estar quietos, sino más 
bien tratar de hacer ejercicio y estar activos porque si no si nos tamos quietos si..." (GF1. P1; 1:6).

\section{DISCUSIÓN}

La percepción que tienen las personas mayores acerca de la estética del cuerpo envejecido se obtuvo con base en la información de los grupos focales realizados para esta investigación. A partir de esto se identificaron diferentes percepciones. Ahora bien, a pesar de que reconocen el envejecimiento como un proceso natural del ciclo vital, este se percibe como un fenómeno en el que se evidencian cambios físicos y psicológicos; pero, a su vez, tratan de mitigarlo con diferentes actividades o prácticas de cuidado y embellecimiento, como lo afirman Yuni et al. (1). Así, la vejez supone la reelaboración y reconfiguración de la identidad, en un proceso que tiene como uno de sus núcleos esenciales la elaboración de los cambios corporales $y$, consecuentemente, el ajuste de la propia imagen corporal. Al mismo tiempo, la vejez como construcción social está relacionada, entre otros, con factores económicos que llevan a considerar esta etapa en función de la productividad y la pérdida de la función y la salud (6).

En ese orden de ideas, el envejecimiento va acompañado de cambios significativos en la apariencia física y la funcionalidad; estas pueden incluir la caída del cabello y el cambio de color, cambios de peso, uso de dentaduras postizas, audición y ayudas para la movilidad y modificaciones en la forma del cuerpo y elasticidad de la piel; sin embargo, la habilidad física es más importante que la apariencia, hecho que coincide con los hallazgos del estudio donde la independencia y la funcionalidad priman en la mayoría de casos sobre la estética en respuesta al deterioro de la salud. No obstante, la imagen corporal es importante desde la identidad, el estatus social y las capacidades (7).

En consecuencia, las personas mayores sienten temor a perder su funcionalidad; por esto, es importante mantenerse en forma, alimentándose bien, arreglándose y adoptando hábitos de buena salud y estilos de vida saludables. Todas las anteriores son prácticas de embellecimiento que ayudan a mitigar los efectos psicológicos de ver la piel arrugada, las manchas y la aparición de otras enfermedades que afectan la forma física del cuerpo directa o indirectamente y lo hacen un actor competente, capaz de tomar decisiones y asegurarse la posibilidad de gozar de su vida en plenitud.

Otra consecuencia que trae la vejez radica en la calidad de vida. Esta se asocia con la pérdida de funciones, lo que hace que su vida se vea sin sentido. Para las mujeres, la apariencia externa es más importante que para los hombres, y se considera útil en el momento de demostrar a los demás que 
es funcional, tanto social como mentalmente. Sin embargo, la capacidad funcional es determinante al fortalecer la autoimagen y la autoestima (8); por ello, los diferentes modos de encarar la vida en la vejez, la manera en que los sujetos se perciben a sí mismos, las estrategias con que luchan por enfrentar sus limitaciones, la aceptación de lo inevitable de ciertos cambios, la disposición a actuar en función de las posibilidades de desarrollo, el deseo de proyectarse y de seguir otorgándole un sentido a la existencia (9) son lo que, finalmente, puede permitir reconocerse como una persona capaz de afrontar los retos del envejecimiento y asumir nuevos desafíos a partir del reconocimiento de sus posibilidades, que hagan que su vida tenga sentido, el cual empieza a reaparecer en el momento que son involucrados en grupos de referencia, puesto que está demostrado que "los viejos suelen estar mejor integrados en aquellas comunidades pequeñas donde no se ha modificado la convivencia de varias generaciones y han permanecido más apartadas del panorama ideológico más globalizante" (9).

Ahora bien, las prácticas de autocuidado, conformadas para esta investigación por las categorías de autocuidado y actividad física y deporte, se desarrollan a través de los grupos de referencia o comunidades pequeñas, que demuestran que el proceso de envejecimiento es un tiempo de transiciones y cambios y que es necesario hacer constantes ajustes adaptativos (1). Adicionalmente, la autonomía funcional es determinante para la autoestima, lo que incide en la imagen corporal (10), pues la autonomía se asocia, además, con el nivel de escolaridad, el estado civil, el estrato socioeconómico y la presencia de alteraciones del sistema músculo esquelético (11).

Hoy en día, la persona mayor se preocupa más por su salud, su forma física, su autocuidado; de esta manera, empieza a repensar sus hábitos alimenticios y de vida, haciendo uso de las dietas y la práctica de ejercicio, para verse y sentirse bien a pesar de su edad, aunque la sociedad pretenda que la persona mayor encaje en un concepto creado. Con el paso de los años, este se desdibuja y se reconfigura un nuevo autoconcepto de cuerpo envejecido, creado de acuerdo con cada representación social y la influencia vivida por cada persona mayor.

El ideario de cuerpo perfecto provoca una inhibición adicional en las personas que envejecen, pues si el cuerpo bueno y activo es el joven, lleno de vigor y frescor, con la vejez, entonces, la persona huye del estereotipo $y$, consecuentemente, es considerada inapta para ejercer las actividades corpóreas (12). Y en este aspecto, la insatisfacción con su imagen corporal hace que las personas practiquen actividad física con frecuencia, a fin de perder peso, deseo presente de igual forma en los participantes de Tunja (13), y es donde se identifica que las prácticas como la 
dieta, el ejercicio y de embellecimiento, en su gran mayoría, son realizadas por las mujeres.

Se encontró que para ellas es más importante su estética corporal que para los hombres, lo que puede ser resultado de aspectos culturales y mediáticos (14). Esto responde también a los estereotipos enmarcados en la sociedad, donde la mujer debe ser ejemplo de belleza y de pulcritud, y la belleza se equipara con la juventud (15) y la apariencia, mediante las cuales las mujeres pueden ganar o perder su estatus social desde lo que se espera de su apariencia socialmente, teniendo en cuenta que en la mirada occidental parecer viejo es un riesgo que afecta el capital social (16). Esto es evidente, así mismo, en mujeres surcoreanas, para quienes el cuidado de sí es importante para la vida cotidiana y el mantenimiento del estatus (17). Aunque también se observa que los hombres realizan estas prácticas, pero no son tan determinantes en su vida.

La persona mayor se ve seriamente influenciada por estereotipos de belleza y funcionalidad que, en ocasiones, menguan la autoimagen o autopercepción que tiene el adulto sobre su cuerpo y sus capacidades. Se sienten relegados por sus familias y fluyen sentimientos de improductividad y de ser prescindibles, que no solamente generan afectaciones físicas, sino psicológicas, que pueden desestabilizar la vida de la persona y hacer de su vejez una etapa de la vida no pro- ductiva, en la cual pierden la posibilidad de vivir una vejez plena en todas sus dimensiones. Esto podría estar sustentado en el comportamiento de las tasas de ocupación y participación relacionadas con la falta de una pensión, según los reportes de la Comisión Económica para América Latina y el Caribe (18). Continuando con este organismo, entre 2002 y 2016, la tasa de ocupación en personas mayores de 60 años pasó del 34,2\% al 35,4\%. No obstante, para el 2016 había un $31,7 \%$ de personas mayores que no trabajaban, ni percibían una pensión, proporción alta especialmente para las mujeres (44,3\%) (19).

Es sabido que la actividad física es importante en el proceso de envejecimiento, para ayudar con la calidad de vida y minimizar problemas futuros con la persona mayor (20). Actualmente, pueden recurrir a diferentes actividades que les permiten continuar con sus vidas, sentirse saludables, aceptar los cambios corporales que implica la vejez, consolidar de nuevo su autoimagen y ser productivos, dado que la insatisfacción con su imagen corporal hace que las personas practiquen actividad física con frecuencia.

Los adultos objeto de la investigación, en su mayoría, recurren a prácticas saludables, para mitigar los rigores de la vejez, lo cual es una experiencia cotidiana que no se debe asociar necesariamente con un estereotipo de belleza o funcionalidad, sino con un hábito que se establece para 
garantizar por largo tiempo la salud y el bienestar físico y mental, sin desconocer que las presiones sobre la imagen corporal se mantienen en esta etapa de la vida y pueden variar según el sexo, el estado civil, la estación, la ubicación urbana/rural, entre otras (21). Esto es lo que permitirá que realmente las personas mayores puedan disfrutar de una adecuada calidad de vida que implica prácticas que promuevan la salud integral, una vida social y familiar activa, la práctica de actividades que le agraden y le ayuden a hacer un uso adecuado de su tiempo, un entorno cálido y afectivo para facilitar la convivencia y la aceptación de las limitaciones propias de la edad entre otras muchas opciones, recordando las implicaciones que los discursos antienvejecimiento traen sobre la imagen corporal y que llevan a una insatisfacción con esta, sobre todo en mujeres (22).

\section{CONFLICTO DE INTERESES}

No se declaran conflictos de intereses.

\section{FINANCIACIÓN}

Investigación financiada por la Universidad de Boyacá.

\section{REFERENCIAS}

1. Yuni JA, Ariel Urbano C, Arce M del C. Discursos sociales sobre el cuerpo, la estética y el envejecimiento. Córdoba (Arg.): Brujas; 2003.
2. Ruiz Calvente M. El cuerpo humano como objeto estético. A Parte Rei Rev Filos. 2010;72:1-7.

3. Han B-C. La salvación de lo bello. Buenos Aires: Herder; 2015.

4. Duque Duque M del P. Representaciones sociales de role de género en la vejez: una comparación transcultural. Rev Latinoam Psicol. 2002;34(1-2):95-106.

5. González J, Fuente R. Desarrollo humano en la vejez: un envejecimiento óptimo desde los cuatro componentes del ser humano. Int J Dev Educ Psychol. 2014;7(1):121-30. https://doi. org/10.17060/ijodaep.2014.n1.v7.783

6. Kehl S, Fernández M. La construcción social de la vejez. Cuad Trab Soc. 2001;14:125-61.

7. Jankowski GS, Diedrichs PC, Williamson H, Christopher G, Harcourt D. Looking age-appropriate while growing old gracefully: a qualitative study of ageing and body image among older adults. J Health Psychol. 2016;21(4):550-61. https://doi.org/10.1177/1359105314531468

8. Fougner $M$, Bergland $A$, Lund $A$, Debesay J. Aging and exercise: perceptions of the active lived-body. Physiother Theory Pract. 2019;35(7):651-62. 
9. Sánchez LM, Monchietti A. Envejecer y parecer joven : obstáculos y consecuencias. Rev Kairós Gerontol. 2013;16(2):9-19.

10. Guerrero Martelo M, Galván G, Vásquez de la Hoz F, Lázaro G, Morales D. Relación entre autoestima y autonomía funcional en función del apoyo social en adultos institucionalizados de la tercera edad. Psicogente. 2015;18(34):30310. https://doi.org/10.17081/psico.18.34.506

11. Quino Ávila AC. Factores de riesgo asociados al nivel de autonomía e independencia en personas mayores, Tunja 2015. Rev Investig en Salud Univ Boyacá. 2018;5(2):259-76. https:// doi.org/10.24267/23897325.322

12. Freitas MC De, Ferreira MA. Vejez y persona anciana: representaciones sociales de adolescentes escolares. Rev Lat Am Enfermagem. 2013;21(3):1-8.

13. Pontrémoli Costa VR, Etchepare Daronco LS, Dias Lopes LF, Gassen Balsan LA. Percepção da imagem corporal de indivíduos adultos e idosos. Rev Bras Obesidade Nutr e Emagrecimento. 2018;2(12):588-96.

14. Sarabia Cobo CM. La imagen corporal en los ancianos. estudio descriptivo. Gerokomos. 2012;23(1):15-8. https://doi.org/10.4321/ S1134-928X2012000100003
15. Macla E, Dial FB, Montepare JM, Hane F, Duboz P. Ageing and the body: one African perspective. Ageing Soc. 2019;39(4):815-35. https://doi.org/10.1017/S0144686X17001313

16. Clarke LH. Women, aging, and beauty culture: navigating the social perils of looking old. Generations. 2017;41(4):104-8.

17. Elfving-Hwang J. Media, cosmetic surgery and aspirational beauty aesthetics of the ageing body in South Korea. Asian Stud Rev. 2020;00(00):1-15. https://doi.org/10.1080/10 357823.2020.1736513

18. Comisión Económica para América Latina y el Caribe (Cepal). Coyuntura laboral en América Latina y el Caribe: la inmigración laboral en América Latina. Washington: Cepal-OIT; 2017.

19. Comisión Económica para América Latina y el Caribe (Cepal). La inserción laboral de las personas mayores en América Latina [internet]. Washington; 2018. Disponible en: https:// www.cepal.org/es/enfoques/la-insercion-laboral-personas-mayores-america-latina

20. De Brito E, Da Cas Prade L, Siqueira LQ, Etchepare Daronco LS, de Paula Leiria M, Valente WP. Flexibilidade, imagem corporal e índice de massa corporal de idosas praticantes de alongamento no Centro Desportivo Munici- 
pal (CDM) Santa Maria/2015. Rev Salusvita. 2016;35(4):477-87.

21. Sánchez-Cabrero $R$, León-Mejía $A C$, Arigita-García A, Maganto-Mateo C. Improvement of body satisfaction in older people: an experimental study. Front Psychol. 2019;10(December):1-11. https://doi.org/10.3389/ fpsyg.2019.02823

22. Cameron E, Ward P, Mandville-Anstey SA, Coombs $A$. The female aging body: a systematic review of female perspectives on aging, health, and body image. J Women Aging. 2019;31(1):3-17. https://doi.org/10.1080/089 52841.2018.1449586

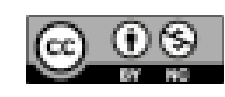

Esta obra está bajo una licencia de Creative Commons Reconocimiento-NoComercial 4.0 Internacional 